\title{
Myelin-Associated Glycoprotein Antibody
}

National Cancer Institute

\section{Source}

National Cancer Institute. Myelin-Associated Glycoprotein Antibody. NCI Thesaurus.

Code C121771.

An autoantibody directed against myelin-associated glycoprotein (MAG). It is associated with active demyelination in peripheral neuropathy and is detected in about $50 \%$ of patients with Ig M paraproteinemia and may occur in some patients with multiple sclerosis, Guillain-Barre syndrome, chronic polyneuropathy, or myasthenia gravis. 